\title{
Customising the Interaction with Configuration Systems ${ }^{\star}$
}

\author{
L. Ardissono ${ }^{1}$, A. Goy ${ }^{1}$, M. Holland ${ }^{2}$, G. Petrone ${ }^{1}$, and R. Schäfer ${ }^{3}$ \\ 1 Dipartimento di Informatica, Università di Torino, Italy \\ \{liliana, goy, giovanna\}@di.unito.it \\ 2 BTExact Technologies, UK \\ matthew.holland@bt.com \\ 3 DFKI. Germany \\ Ralph.Schaeferer77.de
}

\begin{abstract}
This paper presents an intelligent user interface for the management of personalised configuration in business-oriented domains. The proposed system fills the gap between the technical interaction style adopted by current configuration systems and the user's needs, by assisting the user during the selection of the features of the items to be configured and by customising the presentation of the solutions.
\end{abstract}

\section{Introduction}

The techniques currently applied in recommender systems [4] do not support the configuration of items, which is essential to comply with the customer's requirements when purchasing complex products, or registering for services. Indeed, efficient configuration systems have been developed, e.g., see [5], but they require that the user knows all the details about the items to be configured. One way to enhance the usability of such systems is to extend them with user-adaptive interfaces guiding the user through the configuration process.

Within the CAWICOMS project, we developed an intelligent user interface (the CAWICOMS frontend) which mediates the interaction between configuration systems and their users by assisting the user during the specification of the features of the items to be configured and by presenting the solutions in accordance with the user's interests and expertise [2]. This paper presents the CAWICOMS frontend and describes its exploitation in the configuration of IP-based Virtual Private Networks (IP-VPNs).

In order to suitably design the system, we have taken into account a set of requirements which we have collected by interviewing people regularly using the configuration systems available to a telecommunication company and occasional users of on-line configuration systems. In particular, such users complained that the configuration process may require the specification of a large set of data and that this task may be difficult for the non-expert user. Moreover, they pointed out that the configuration of items and the presentation of solutions should be organised by taking the structure of the items into

\footnotetext{
${ }^{\star}$ Copyright by Springer Verlag. This paper is going to appear on the proceedings of the 9yh International Conference on User Modeling, 2003
} 
account, and by focusing on the most interesting features for the user, instead of being specified as a flat feature list, as most configuration systems do. The users also had interaction requirements concerning the possibility to postpone configuration decisions and to trigger the automatic configuration of items. ${ }^{1}$

\section{Adaptive User Interaction}

The CAWICOMS frontend manages the interaction with the user as a dynamically generated sequence of configuration steps, where the user is asked to set the features of the item to be configured. The configuration solutions are presented by focusing the descriptions on the features fitting the user's interests and expertise. ${ }^{2}$ The generation of the user interface is tailored to the user's expertise and interests and relies on an explicit representation of the knowledge about configurable items, on the management of a dynamic user model describing the end-user's features, and on the application of adaptive hypermedia techniques [1].

\subsection{Representation of Knowledge about Products and Services}

The representation of the knowledge about products and services relies on the componentport approach for configuration described in [5]. To support a user-oriented management of the configuration process, we have extended this representation in various ways. For instance, we enriched the representation of features with an explanation of their meaning and the specification of the type of information they convey (e.g., technical, economic, general information). Moreover, we specified a complexity level representing an estimate of the degree of expertise required to understand the meaning of the feature. Furthermore, we specified a criticality level, needed to identify features whose values should be acknowledged by the user (important features, from the configuration, or business point of view). We also introduced the representation of properties aimed at evaluating the items from a qualitative point of view. In the IP-VPN domain, we defined properties such as the performance and the reliability of the networks. The impact of the technical features on the evaluation of the item properties is represented by defining evaluation functions that map property values onto the feature values as defined by Multiattribute Utility Theory [7].

\subsection{Management of the User Model}

An individual user model stores explicit and implicit information about the user. The explicit portion of the user model stores the user's characteristics, such as the nationality and the type of company she represents. The implicit portion describes the system's estimates about the user's interests in the properties of the configurable items (e.g.,

\footnotetext{
${ }^{1}$ We will not address such issues in the present paper, for space reasons.

2 The ILOG JConfigurator engine is used to generate the configuration solutions.
} 


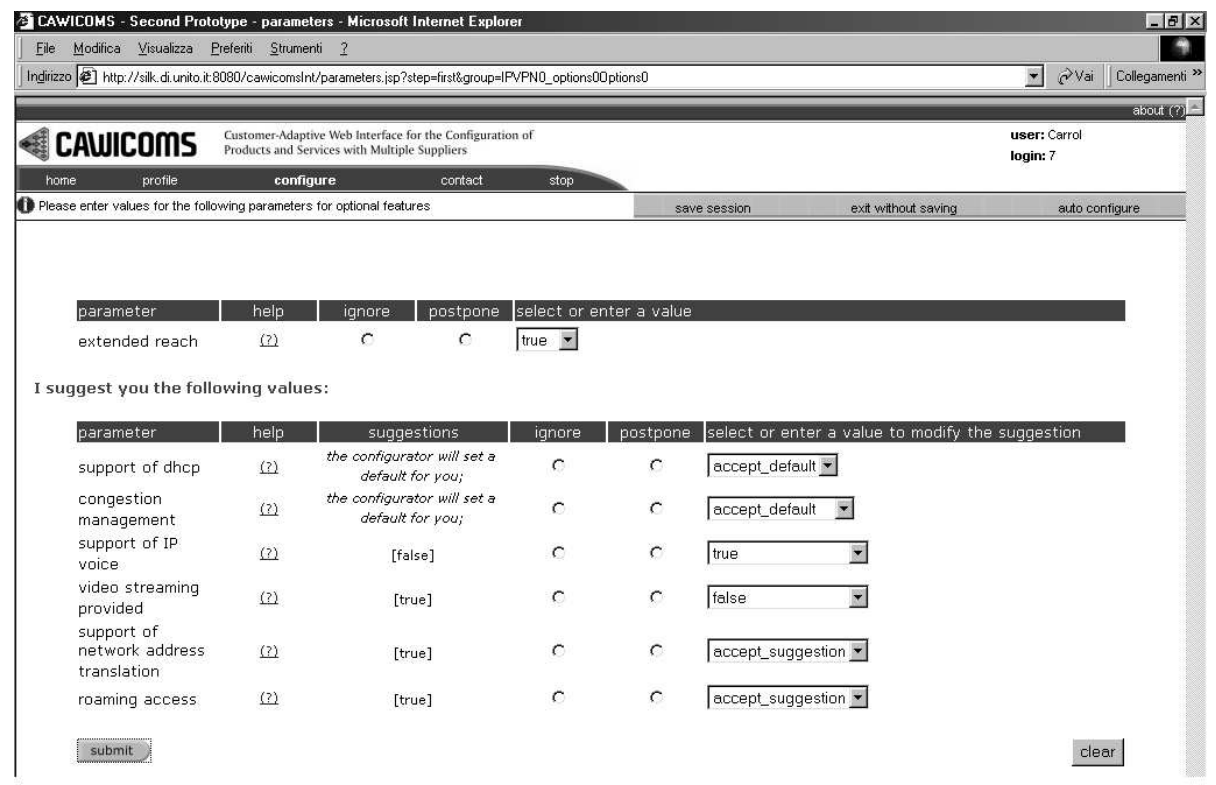

Fig. 1. A step in the configuration of an IP-VPN.

reliability and economy) and the estimates of the user's knowledge about the features of the configurable items. ${ }^{3}$

The user's knowledge and interests are estimated by combining stereotypical information about the classes of users of the system (e.g., technical engineers, managers, end-customers) with user modeling acquisition techniques, based on the interpretation of the user's interaction behaviour. In particular, the system estimates the user's interests in the properties of products and services by ascribing her a model for the evaluation of items based on the Multiattribute Utility Theory. This model defines the evaluation of items as a function of the evaluation of their properties, given the user's interests in such properties. The exploitation of this theory enables the system to interpret the acceptance or the rejection of a specific configuration solution as a sign that the user's evaluation of the item is good/bad. In turn, this information is relevant to assess the user's interests in the item properties. As the interpretation of the user's actions is affected by uncertainty, we use Bayesian Networks to perform this task [6]. Finally, the user's expertise is estimated by applying the approach described in [3].

\subsection{Elicitation of the user's requirements}

The system employs the estimates on the user's current interests in the item's properties, such as its reliability, to steer the configuration process by proposing feature values and

\footnotetext{
${ }^{3}$ As the estimates of the user's interests and expertise are uncertain, they are represented as probability distributions on the values of variables associated to the knowledge of items and to the properties.
} 


\begin{tabular}{l|l|} 
your interest in... & level \\
reliability & low
\end{tabular}

Fig. 2. Elicitation of user requirements about a property of IP-VPNs.

components that maximise the user's expected evaluation of the solution. In this way, the number of features she has to set is dramatically reduced. The system also uses other personalisation strategies. For example, features can be set by applying personalised defaults describing business rules based on the customer's characteristics.

The personalisation strategies are represented as declarative rules with priority. Given a feature to be set, a rule-based engine selects the strategy to be applied by evaluating the alternative rules according to their priority and by choosing the first one matching the current situation in a satisfactory way. The evaluation is performed by taking into account, on the one hand, the complexity and the criticality of the feature, and, on the other hand, the user's expertise and interests in the item properties. The following strategies are defined:

1) Very critical features have to be set by the user.

2) If a personalised default matching the user's characteristics is available for the feature and the suggested values are consistent with the current domain of the feature, suggest the default values.

3) If the feature is related to some properties for which the user's estimated interest is low, set a default value consistent with the domain of the feature.

4) If the user's estimated expertise is sufficient to choose a value for the feature, ask her to set the preferred value, given the current domain.

As asking the user is the last resort, whenever possible, the system suggests the values to be set. Figure 1 shows a page generated during the configuration of the optional services of an IP-VPN. The system asks the user to specify whether the company (a Web Design one) wants the extended reach to Eastern countries. Moreover, the system suggests to set some features in a standard way (support of dhcp, congestion management protocol). Furthermore, personalised defaults are suggested for the support of IP voice, the provision of video streaming, the support for network address translation, and the roaming access. The suggestions can be overridden, as the user did with the IP voice and video streaming. As shown in Figure 2, the system may also elicit the required information in an indirect way, by asking the user about her preferences for properties of an IP-VPN related to the features to be set. Given the answer, the most suitable settings to achieve such properties are determined.

\section{Evaluation}

The prototype system was evaluated a number of times throughout the development process. This evaluation was used to guide the further development of the prototype. In order to test features of the adaptive interface, we used role-playing exercises involving a small number of users with different levels of expertise. Some of them were familiar with existing configuration systems, but not necessarily IP-VPN, some were familiar 
with the domain, some completely new to this area of technology. These users gave feedback in the form of a questionnaire.

We performed tests using two sets of scenarios: One for the Telecommunications Switches domain and one for the IP-VPN domain. Here, we concentrate on the IP-VPN domain, which was used for the final evaluation. Our IP-VPN test scenarios involved a fictitious company named WooCorp. WooCorp are a fast growing technology start-up company with offices across Europe and need to purchase a VPN to replace their existing collection of ad-hoc communications links. They have decided to go to a reseller (Network Global Services) who employ a CAWICOMS configuration system. We developed a number of different test scripts within this scenario going from the initial specification of a small network to connect WooCorp's major sites, then adding in more complexity such as further sites and dial-up access for mobile users.

The results of this evaluation were quite satisfactory. The total number of questionnaires analysed was 30. Approximately half of the test subjects were novices, with $10 \%$ in the "Intermediate" bracket. Our test users particularly liked the features designed to help novice users: $55 \%$ liked the suggestion functionality and 35\% explicitly praised the auto-configure feature. This feature was equally liked by novice and expert users. $70 \%$ of users felt that the adaptive features of the system improved the user experience. As for future development of the system, it was suggested that the adaptive features could go even further: expert users thought that explanations of why particular values were suggested would aid them.

\section{Conclusions}

We have presented the intelligent user interface employed in the CAWICOMS configuration system to assist the user during the configuration of complex services. This user interface is based on the dynamic generation of the Web pages, which are personalised by taking the user's expertise and interests into account.

CAWICOMS is the acronym for "Customer-Adaptive Web Interface for the Configuration of Products and Services with Multiple Suppliers"; see http://www.cawicoms.org. This work was funded by the EU through the IST Programme under contract IST-1999-10688. We thank the other project partners (Telecom Italia Lab, ETIS, ILOG SA, University of Klagenfurt) for their contribution to the design and development of CAWICOMS.

\section{References}

1. The adaptive Web. Communications of the ACM, 45(5), 2002.

2. L. Ardissono, A. Felfernig, G. Friedrich, A. Goy, D. Jannach, M. Meyer, G. Petrone, R. Schäfer, W. Schütz, and M. Zanker. Personalising on-line configuration of products and services. In Proc. 15th Conf. ECAI, pages 225-229, Lyon, 2002.

3. A. Jameson. Knowing What Others Know: Studies in Intuitive Psychometrics. PhD thesis, University of Amsterdam, 1990.

4. A. Kobsa, J. Koenemann, and W. Pohl. Personalized hypermedia presentation techniques for improving online customer relationships. The Knowledge Engineering Review, 16(2):111$155,2001$. 
5. D. Mailharro. A classification and constraint-based framework for configuration. AI in Engineering, Design and Manucturing, 12:383-397, 1998.

6. W. Schütz and R. Schäfer. Bayesian networks for estimating the user's interests in the context of a configuration task. In R. Schäfer, M. E. Müller, and S. A. Macskassy, editors, Proc. UM2001 Workshop on Machine Learning for User Modeling, pages 23-36, July 2001.

7. D. von Winterfeldt and W. Edwards. Decision Analysis and Behavioral Research. Cambridge University Press, Cambridge, UK, 1986. 